\title{
Impact of Signal Energy from Vedic Chanting on Human Neurological System
}

\author{
Veera Raghava Swamy Nalluri, Vj.k. Kishor Sonti, Sundari.g
}

\begin{abstract}
The human neurological system has an effective impact by meditation, mantra therapy, music therapy, Raga therapy and Vedic chanting. To detect this effect on Human Neurological system, it is necessary to analyse the signal energy from the appropriate sources which are generating audible frequencies. By these generated frequencies, the Central Nervous System of brain will be relaxed. This process follows, Calculation of Special acoustic signals originated from Vedic Chanting, classification of these signals with respect to the levels of amplitude and frequency, analysing the impact of these signal energies on the neurotransmission. It also focuses and involves limbic and para-limbic regions of neuro-transmission. This paper presents impact of vedic chanting signal positive vibrations on human body brain and nervous System.
\end{abstract}

Keywords: Vedic chanting, Neurotransmission, Limbic Resonance, Homeostasis and Signal energy

\section{INTRODUCTION}

In ancient Sanatana culture spiritually includes Philosophy, Tantra, Yoga, and Agama. These are all parts of knowledge in Vedas. Vedas as authority of Sabda pramana; Sabda alludes to the words verbally expressed as mantras. In the creation procedure, Shabda is the utmost unique element that surrounds the space. The scriptures say 'Sabdaikagunamakasam', which means "Only sound qualifies space". Be that as it may, sound spreads through air, fire, water and earth..Sound as the sole property of space can likewise be seen as far as acoustic nature of space in encased regions. Sabda assumes a significant job known to man made out of five elements. This Sabda or Sound or acoustic sound that generates out by the pronunciation of Vedic text or mantra is believed to have a great influence on our psychic system. Sounds of traditional Vedic chanting produce vibrations those have beneficial spiritual effects both on chanters and listeners.

Six basic aspects of phonetics, which are important to Vedic chanting are Varna, Swara, Mantra, Balam, Sama and Santana, all these six aspects are inter-related. In modern day lifestyle, humans are prone to diverse psychological and physiological issues those are inducing pressure or stress,

Revised Manuscript Received on December 13, 2019.

* Correspondence Author

Veera Raghava Swamy Nalluri*, Research Scholar, Department of Electronics and Communication Engineering, Sathyabama Institute of Science and Technology, Chennai - 600 119, India, raghavaswamynv@gmail.com,

V.j.k. Kishor sonti, Associate Professor, Department of Electronics and Communication Engineering, Sathyabama Institute of Science and Technology, Chennai - 600 119, India, kishoresonti.ece@sathyabama.ac.in

Sundari.G, Professor, Department of Electronics and Communication Engineering, Sathyabama Institute of Science and Technology, Chennai 600119, India which is unwarranted. This obviously disturbs our mental health, where some sort of natural or artificial rejuvenation has become a necessity.

In recent research, neuroscientists have perceived that tuning in to music, mantras and Vedic chanting are an effect on all human cognitive functions. Vedic texts, music or mantras have turned as a precious tool effecting significantly different parts of the human cerebrum. The sound of the Vedic chanting is sacred because not only their physical characteristics have deeper phonetic influence but also due to their impact on the listener's mind. Vedic chanting also primes our neural pathways toward connection and safety, body and brain and also aligning breath.

This study exactly refers to such practices that researchers are exploring in the recent past and our research focal point is to revisit and represent such practices with more authenticity through experiments. This paper rightly explores all the aspects related to this novel study and brings out some good work of earlier researchers and tries to draw logical pathways for the future generations, who are passionate to work in this domain.

\section{VEDIC HYMNS OR MANTRAS - SIGNIFICANCE IN PSYCHIC PERSPECTIVE}

The Vedas planned explicit hymns with explicit zones of influence. The singing of these hymns had specific frequencies, and performed homas explicitly structured all the time. Vedic melodies produce incredible sound vibrations that are fit for changing vitality at all degrees of development. They influence the psychological and arrive at the subliminal level where our karmic patterns are stored. The act of singing builds memory, expands fixation and deletes the impacts of past karma. The best possible recitation of mantras can diminish stress and pressure.

Each sound has a distinct vibration, as well as, each chant has a different effect. Each chanting is designed to have a distinct vibrating energy. The chanting of the Veda mantras have three aspects namely; purity of mind, purity of intonation and purity of pronunciation. Sound of chants can carry the listeners to spiritual experiences. The sounds of the Vedas are sacred because not only their physical characteristics have deeper phonetic influence on the listener's mind.

In ancient sanathana mythology, it was believed that "Maharishi" knows the benefit of Mantras and their proper pronunciation. The effect is beyond human imagination and difficult to present in qualitative manner. 
But with the advent of signal energy measuring techniques, researchers could able to advance in this perspective. Present day science, psychology and spirituality are interlaced in such a way that the interdependency is well prevalent due to various factors. In some parts of the globe, it is highly in practice and in belief that chanting Vedic text not only rejuvenates the mind but also clear the sins that a human commits in a lifetime.

\section{IMPACT ANALYSIS OF SIGNAL ENERGIES FOR DIFFERENT FREQUENCIES IN AUDIBLE RANGE}

Spiritual attributes of this work becomes less significant and not associated to particular belief or practice, when science comes into intervention. Studies proven that "Hatayoga" to "Himalayan Meditation" practices yielded wonderful results in understanding our mind and body balance.

Signal is that which conveys information, the acoustic signal is that which explains the information in most understandable format to human mind. The Mantra, eventually an acoustic signal is a combination of sound, breath and rhythm. This surprisingly, channelizes human biological energy system. "Omkara" chanting and other vedic verses had profound influences on all the energy centres of the human body. This argument is further strengthened by the 'CHAKRAS' science that there are 7 energy centres in human body, which can be tapped with proper rhythm of breath that is in turn shall be controlled with proper pronunciation of certain frequencies of acoustic energy.

According to Science, the human body has "Self-regulating" system known as homeostasis to maintain the stability of body parameters. The energies emerging from vedicchanting impact the medulla, the sound-related nerve that associates with every one of the muscles of the body through neurotransmission. These vibrations are through the vagus nerve, the internal ear interfaces with the larynx, lungs, heart, liver, stomach, kidneys, bladder, little and digestive organ. The researchers derived that the Vedic writings are possible to declare the readiness of the neurological structure. The FMRI study report assumes and recommended that melodic familiarities are identified with limbic, paralympic and reward circuits [23]. Evidences of earlier investigations showed that specific degrees of desire and consistency brought are due to excitement generated out of experiencing music. [24].

\section{FINDINGS AND OUTCOMES OF EARLIER RESEARCH}

In 2004, Jina Devi et.al [2-3] has conducted a study on analyzing of Vedic mantra "Omkara" and digital Spectral analysis of alphabets A, U, Ma. "OM" for male and female voice with time spectra grams was studied. The observations suggested that the male voices have a more number of subhormonics than the female voices.
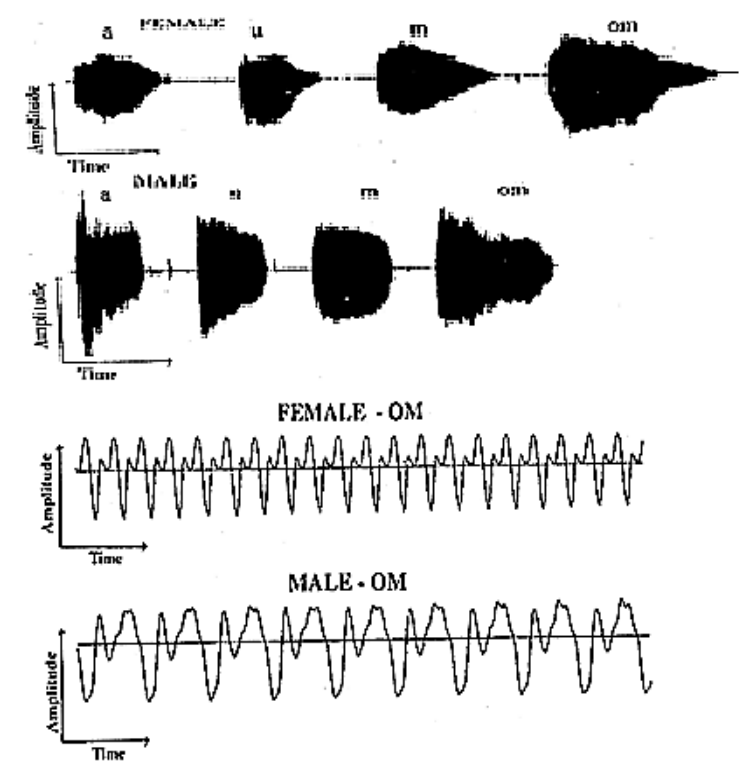

Fig. 1. Digital Spectal Analysis For Male And Female Voice Of 'OM' [2-3]

In other experiment by Sripadghaligi,et.al[4] during 2006 , the effect of vedic chanting on memory and sustained attention was studied. A pre selected cross-sectional study on chanting and non-chanting groups was carried out for two years. It was observed that, when letter and character test was conducted chanting group showed better verbal and special scoring than non-chanting group.

Ajay A Gurjar and Sidhartha L. et al [5],[6] during 2008 and 2016 performed FFT analysis of power versus frequency of the signal energies associated with OM. The findings suggested that OM shall be considered as a cosmic sound and the totality of all sounds. The interesting finding is the deviations in Electro Encephalograms (EEG) waves towards low frequency rhythms of OM chanting.

The inferences were also drawn about these changes may be due to electrical activity of the brain produced under the influence of this vedic chanting. In this work, Timefrequency analysis is performed on OM mantra signal. They utilized wavelet transforms for the Time- frequency analysis. In this research work they attempt to analyze the sound characteristics of OM by using wavelets and MATLAB 7.0 has been used for the analysis. The procedure followed in their research is depicted in figure.2.

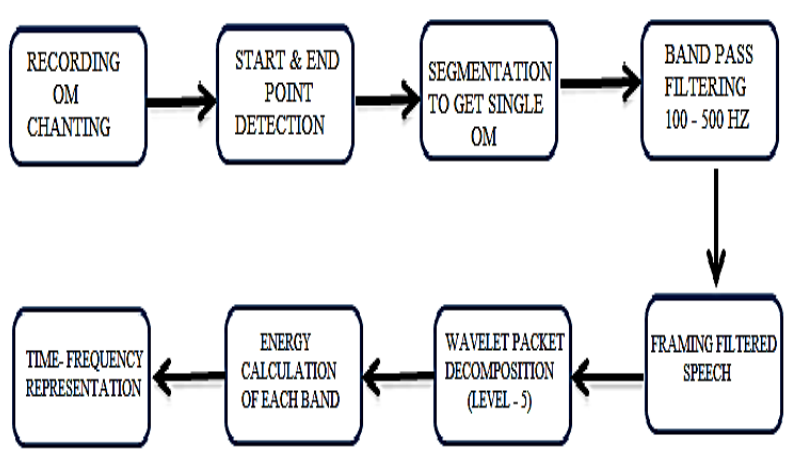

Fig.2. Wavelet Packed Based Analysis [5-6] 
There is another interesting study by Kumar SaiSailesh et.al [11] in 2016 on analyzing of 'Beneficial effect of 12 weeks of Sri Vishnu sahasranama chanting. He observed the effectiveness of this chanting for stress management. The method has chanting of vishusahasranama every day for 12 weeks. DASS-42 (Depression, Anxiety and stress Scale) and Serum cortisol test was used to assess stress, blood pressure was recorded to assess automatic functions and spatial, verbal memory test were used to assess cognitive functions. All outcomes before and after 12 weeks were recorded and found a significant deviation in reduced stress levels were observed after being prone to the consistent listening of this vedic chanting.

In 2017, Anita Padam,et.al [12] conducted an investigation on the impact of tuning in to Vedic tunes and old style Indian instrumental music in patients experiencing upper stomach related endoscopy is another interesting experiment. In the same year, Balaram P. et.al [13] studied impact of Gayatri Mantra and poem recitation methods using the Digit Letter Substitution Task (DLST) method. The conclusion of this study is Gayatri Mantra Chanting produced better results than Poem recitation. The qualitative data obtained in this study is quoted here for the reference in figure.3. [12].

\begin{tabular}{|c|c|c|c|c|c|}
\hline \multirow{3}{*}{$\begin{array}{l}\text { GROUP } \\
\text { Whole } \\
(\mathrm{n}=60)\end{array}$} & \multirow[t]{2}{*}{ SCORE } & \multicolumn{2}{|c|}{ PL CHANTING } & \multicolumn{2}{|c|}{ GMCHANTING } \\
\hline & & PRE & POST & PRE & POST \\
\hline & Total & $47.62 \pm 12.62$ & $50.88 \pm 12.71^{\text {ニ* }}$ & $46.67 \pm 13.03$ & $56.25 \pm 12.37$ \\
\hline & Wrong & $1.20 \pm 0.29$ & $1.43 \pm 0.17$ & $0.80 \pm 0.27$ & $1.77 \pm 0.12^{* * *}$ \\
\hline & Net & $46.07 \pm 13.43$ & $49.43 \pm 12.73^{* *}$ & $45.70 \pm 12.70$ & $\begin{array}{c}54.83 \pm \\
12.39^{* * *}\end{array}$ \\
\hline Male $(n=30)$ & Total & $44.7 \pm 11.61$ & $50.3 \pm 14.33^{* *}$ & $49.5 \pm 12.03$ & $\begin{array}{c}58.6 \pm \\
15.78^{* * * *}\end{array}$ \\
\hline & Wrong & $1.27 \pm 1.36$ & $2.4 \pm 2.65^{*}$ & $1.1 \pm 1.69$ & $3.3 \pm 3.01^{* * *}$ \\
\hline & Net & $42.73 \pm 10.62$ & $47.87 \pm 12.5^{* * *}$ & $48.07 \pm 11.56$ & $56 \pm 14.04 * * *$ \\
\hline $\begin{array}{c}\text { Female } \\
(\mathbf{n}=\mathbf{3 0})\end{array}$ & Total & $50.53 \pm 11.4$ & $51.47 \pm 9.57$ & $43.83 \pm 11.77$ & $\begin{array}{c}53.9 \pm \\
10.74^{* * * *}\end{array}$ \\
\hline & Wrong & $1.13 \pm 1.55$ & $0.47 \pm 0.82$ & $0.5 \pm 0.86$ & $0.23 \pm 0.73^{*}$ \\
\hline & Net & $49.4 \pm 10.7$ & $51 \pm 9.51$ & $43.33 \pm 11.62$ & $\begin{array}{c}53.67 \pm \\
10.57^{* * *} \\
\end{array}$ \\
\hline
\end{tabular}

\section{Fig.3. The Qualitative Analysis Carried Out By Anita Padam Et.Al [12] On Vedic Text Chanting}

JyotiKori et.al in 2017 conducted one more experiment on impact of OM reciting on the heart beat rate. In this test, twenty members were approached to sing OM for five minutes per day for about a month. His heartbeat was recorded when singing [14]. They analysed frequency of OM mantra. The Power Spectral Density (PSD) of OM was recorded and studied. It was inferred that the focussed OM chanting or listening can dispose of undesirable thoughts from the psyche and make the mind quiet down thereby limit the heart beat rate.

In another study of 2016, Priya P. R., Kumar S S et.al [15] done experiment on women with Hypertension. The process followed in this experiment is: Initially, participants underwent practice Om NamahShivaya chanting sessions for 3 days under supervision of yoga teacher. After that they were requested to chant Om NamahShivaya for 108 times in the morning for 40 days. All parameters were collected at 9.00 A.M. to prevent diurnal variations. Demographic data was presented in this study by the researchers and observed a significant decrease in depression, anxiety, stress scores, cortisol levels.

In a few different examinations led by various analysts [16-25] on melodic perception, researchers explored the effect of nature with the activity of the electroencephalogram-subordinate cerebrum (EEG). The significant emotion recognition based on EEG was recently proposed by the researchers with the high temporal resolution prominence of EEG with low cost.

\section{CONCLUSION}

Vedic text or Vedic chanting producing distinct signal energy such as Music has intense influence on human moods and emotions. The origin of mantras or vedic text or vedic hymns shall be observed from Vedas of Sanathana tradition. The studies inferred that chanting mantras will create vibratory sensation around the ears and these sensations are transmitted through branches of vagus nerve. Influence on limbic areas, amygdala, hippocampus and thalamus was also observed by vedic chanting.

The inferences based on various studies suggest that, there is a high degree of necessity to find the methods to reduce increasing stress levels or mood variations in humans. Of course, music is one already explored method, but the interest and institution to scientific community is slowly increasing on the Mantras Chanting and their impact analysis on human neurological systems.

The studies shall be extended to various other control parameters of experiments such as age, gender, cultural background and intensity, frequency of the vedic verses. The scope also lies to various other forms of vedic literatures as earlier studies restricted for unknown reasons only to certain specific chartings such as OM. This review paper focussed on exploring the pace and progress of this much needed research problem for the societal benefit and also presented the grey areas to work for future enthusiastic learners.

\section{REFERENCES}

1. M. Khan and A. Ajmal; " Effect of Classical and Pop Music on Mood and Performance”; ISSN 2250-3153;12;Dec 2017.IJSRP Volume 7, Issue 12, 2017

2. Anita P., et.al, 2017 conducted a study of "Effect of listening to Vedic chants and Indian classical instrumental music on patients undergoing upper gastrointestinal endoscopy" in2017 Indian Journal of Psychiatry

3. JyotiKori et.al (2017) "Effect of chanting OMKARA mantra on pulse rate for stress reduction" IJERD 2017

4. Balaram P., S. GodseDerle et.al (2017) "Comparison of effect of Gayatri Mantra and Poem Chanting on Digit Letter SubstitutionTask Ancient science of life", oct-dec 2012/Volume 32/Issue 2

5. K. SaiSailesh et.al (2016) conducted a study on analyzing of "Beneficial effect of 12 weeks Sri Vishnu sahasranama chanting on stress, cognition and automatic functions" Int J.Res. Ayurveda Pharm7. (Suppl 4) sep-oct,2016.

6. Kulkarni A Ashok, J Abhijit H,G. NehaD."An understanding towards the mode of action of benfits of mantra chanting" Int. J. Res Ayurveda Pharm, 36 -38. http://dx.doi.org/10.7897/2277-4343.07251,Mar-Apr 2016

7. Ajay Anil G. and Sidhartha A. Ladhake et.al(2016) "Analysis and dissection of Sanskrit Divine sound "OM" using digital signal processing to study the Science behind "OM" Chanting". It is published in 2008 and 2016 7th International Conference on Intelligent Systems, Modelling and male voices Simulation.

8. P. P. Roy, K. SaiSailesh , R.Archana, S.Mishra et.al IJPSR (2016) "Om Namah Shivaya Chanting For Management Of Stress In Elderly Women With Hypertension” IJPSRA 2016, Vol. 7,Issue 11. 
9. Hsieh $\mathrm{S}$, Lee $\mathrm{YY}$, "Classifying different emotional states by means of EEG-based functional connectivity patterns". PLoS One 9(4):e95415. doi:10.1371/journal.pone.0095415, 2014.

10. Oh E, Kim M, Kim SP, Kim MK, "A review on the computational methods for emotional state estimation from the human EEG" Comput Math Methods Med 2013:1-13. doi:10.1155/2013/573734, 2013.

11. Chen HH, Wiley-Blackwell,HobokenYang YH, "Music emotion recognition". CRC Press, BocaRaton, 2011.

12. Argstatter,H. and Mohn,C (2010). "Perception of six basic emotions in music."

13. A. Gurjar\&Sidhartha A. et.al (2009) "Spectral analysis of Sanskrit devine sound OM “, IJT 8(5): 781-785,2009.

14. S.Koelsch, D Sammler, T.Fritz, M.Grigutsch, "Music and emotion: electrophysiological correlates of the processing of pleasant and unpleasant music." Psychophysiology44(2):293-304 doi:10.1111/j.1469-8986.2007.00497.x, 2007.

15. S Koelsch(2012) "Brainandmusic." CRC Press, BocaRaton.

16. J Plailly, B Tillmann, JP Royet, "The feeling of familiarity of music and odors: the same neural signature? " Cereb Cortex 17(11):2650-2658.doi:10.1093/cercor/bhl173, 2007.

17. S. Ghaligi,et.alt(2006) conduct experiment on "Effect of vedic chanting on memory and sustained attention" IJTK, vol.5(2), April 2007, pp. 177-180

18. T.Lesiuk, "The Effect Of Music Listening On Work Performance psychology of music.", vol. 33, 173-199, 2005.

19. JINA DEVI H. et.al " Spectral analysis of Vedic mantra Omkara “ Indian journal of Traditional knowledge , vol.3(2), April 2004, pp.154-161.

20. H Jina Devi, "Concept of Mantra and its corresponding qualities", MS Dissertation, Hindu University of America, Florida, USA, January 2003.

21. Trainor LJ, Schmidt LA, "Frontal brain electrical activity EEG distinguishes valence and intensity of musical emotions". Cogn Emot 15(4):487-500.doi:10.1080/02699930126048, 2001.

22. T.M.Srinivasan and B.G.Sreelakshmi(eds),et.al "Sense perception in science and shastras", Proceedings of the conference in Shankara Mutt,Bangalore,India, 1985.

23. N. Thammasan; "Investigation of Familiarity Effects in Music-Emotion Recognition sBased on EEG".

24. NattapongThammasan K.,Moriyama,Ken-ichiFukui.,Masayuki N. "Investigation of Familiarity Effects in Music-Emotion Recognition Based on EEG" 